\title{
The Causal Efficiency of the Passage of Time
}

\author{
Jiri Benovsky
}

Received: 22 December 2011 / Revised: 22 February 2012 / Accepted: 27 February 2012 /

Published online: 25 May 2012

(C) Springer Science+Business Media B.V. 2012

\begin{abstract}
Does mere passage of time have causal powers? Are properties like "being $n$ days past" causally efficient? A pervasive intuition among metaphysicians seems to be that they don't. Events and/or objects change, and they cause or are caused by other events and/or objects; but one does not see how just the mere passage of time could cause any difference in the world. In this paper, I shall discuss a case where it seems that mere passage of time does have causal powers: Sydney Shoemaker's (1969) possible world where temporal vacua (allegedly) take place. I shall argue that Shoemaker's thought-experiment doesn't really aim at teaching us that there can be time without change, but rather that if such a scenario is plausible at all (as I think it is) it provides us with good reasons to think that mere passage of time can be directly causally efficient.
\end{abstract}

Keywords Passage of time $\cdot$ Causality

\section{$\S 1$}

Does mere passage of time have causal powers? Are properties like "being $n$ days past" causally efficient? A pervasive intuition among metaphysicians seems to be that they don't. Events and/or objects change, and they cause or are caused by other events and/or objects; but one does not see how just the mere passage of time could cause any difference in the world. A slice of pizza that has been in the fridge for too long caused me to have stomach ache. It was first fresh, then rotten. Such qualitative changes, the intuition goes, as all changes in the world, are due to causal processes other than the mere fact that some time elapsed between the moment the slice was put in the fridge and the moment I ate it - the property of the slice of "being 19 days old" is not, in any way, directly and by itself causally responsible for the state of the pizza

J. Benovsky $(\bowtie)$

Department of Philosophy, The University of Fribourg, Fribourg, Switzerland

e-mail: jiri@benovsky.com

URL: www.jiribenovsky.org 
after 19 days in the fridge; rather, such changes, as all changes in the world, involve a causal story about other properties, like properties involving the number of bacteria that increased over time. The passage of time, of course, plays a role in the decay of the slice of pizza, but it is not the passage of time itself and alone that caused it to be anything, rotten or otherwise.

In this paper, I shall discuss a case where it seems that mere passage of time does have causal powers: Sydney Shoemaker's (1969) possible world where temporal vacua (allegedly) take place. I shall argue that Shoemaker's thought-experiment doesn't really aim at teaching us that there can be time without change, but that if such a scenario is plausible at all (as I think it is) it rather provides us with good reasons to think that mere passage of time can be directly causally efficient.

\section{$\S 2$}

Let me briefly summarize Shoemaker's well-known thought experiment. Shoemaker himself intended it to show that there can be periods of time-called 'global freezes'-when all changes in the universe come to a stop, while time itself continues to flow. More precisely, the purpose of his argument is to show that in a possible world where 'local freezes' occur, its inhabitants can have a good reason to think that 'global freezes' occur (even if, of course, no one can directly experience them). Take a world $\mathrm{W}$ divided into three spatial zones A, B, and C. There are local freezes : at regular intervals, in each of the zones all changes come to a stop for a period of time, while at least one of the other two zones remains unfrozen. This happens for $1 \mathrm{~h}$ every 2 years in zone A, for $1 \mathrm{~h}$ every 3 years in zone B, and for $1 \mathrm{~h}$ every 5 years in zone $\mathrm{C}$. Thus, the inhabitants of $\mathrm{W}$ who can be aware of local freezes when they occur in a different zone than the one they are located in, and who have made the proper calculation, have a good reason to believe that every 30 years there is a $1 \mathrm{~h}$ global freeze, where change stops in all three zones. To make their evidence stronger, the thought-experiment can be enriched by adding that in every zone just 1 min before a local freeze occurs some visible changes occur to 'announce' the forthcoming freeze: for instance, just before a local freeze occurs all things located in the zone in question turn red. Every 30 years, it is then not only a simpler theory (it is simpler to say that local freezes occur with a regularity rather than to say that there is an exception in the regularity every 30 years) but also the fact that everything, in all zones, turns red $1 \mathrm{~min}$ before the expected global freeze, that indicate that indeed such a global freeze is about to take place. It is thus, in $\mathrm{W}$, reasonable to believe that there are regular $1 \mathrm{~h}$ periods where time continues to flow while no change at all occurs.

Even from this brief summary of Shoemaker's argument it is clear that it doesn't show that there can be global freezes - temporal vacua. First, because the possibility of local freezes is just presupposed without argument; second, because such a presupposition carries with it a number of difficulties (see for instance Warmbrod (2004) for a recent detailed criticism of Shoemaker's argument); and third, because even if these difficulties could be overcome, the most that can be learned from this thought-experiment is that in a world like $\mathrm{W}$ its inhabitants would have good reasons to believe in temporal vacua - an epistemological claim. The metaphysical possibility of temporal vacua is then mostly untouched by Shoemaker's argument : one could 
very well argue that even if local freezes are possible, global freezes aren't, precisely because they are global, and because there can be no time without change, and Shoemaker's thought-experiment simply isn't designed to deal with that. Furthermore, quite importantly, the difficulties that are often raised against Shoemaker's thought-experiment attack precisely the-epistemologically loaded, and thepossibility-of-local-freezes-related-way in which Shoemaker developed it.

For instance, in the case of local freezes, as described by Shoemaker, a lot of adjustments of physical laws would need to be made, since for instance a photon travelling towards a frozen region of $\mathrm{W}$ from an unfrozen one could not enter it and would somehow have to be reflected back or stopped; while of course the frozen region itself could not emit any photons since that would require movement (thus, change). Consequently, it could actually not be observed as being a frozen region at all, rather, to the observers from the unfrozen regions, the frozen one would look as if it had simply disappeared. Other interactions, like gravitational forces or electromagnetic forces would also need to cease to be efficient between a frozen region and an unfrozen one, thus causing important gravitational, or other, shifts in the unfrozen part of W. Also, people or objects standing at the frontier between the two zones right at the moment when a local freeze starts would see a half of them disappear but without really ceasing to exist. (I do not intend these quick comments to conclusively refute the metaphysical possibility of local freezes, but only to indicate the kind of problems one would have to deal with if one wanted to develop a full-fleshed defence of such a possibility.)

Among epistemological difficulties, discussed by Shoemaker himself, there is the one of defending the view that for the inhabitants of $\mathrm{W}$ it is more reasonable to believe in the existence of a global freeze every 30 years, based on statistical extrapolation from the frequency of occurrences of local freezes, than to believe in the fact that every 30 years there is an exception in the regularity of local freezesperhaps precisely because they believe that global freezes are not possible (especially if they interpret locally frozen regions not as being frozen but as having disappeared). Shoemaker claims that inhabitants of $\mathrm{W}$ would have a good reason the prefer the first hypothesis over the second, namely its simplicity. Indeed, it would require a more complicated (ir)regularity law to account for exceptions in the succession of local freezes that would always avoid global ones (for example, local freezes in region A would happen every 2 years except for the 30th year, and so on). According to Shoemaker, it would then be simpler, and consequently more reasonable, to avoid believing in such exceptions. But here, issues about simplicity arise: is it more complicated to believe in a more complicated regularity law or is it more complicated to believe in the possibility of time without change? One kind of simplicity is mathematical simplicity (equations predicting the behaviour of local freezes would need to be more complicated (but, actually, not very complicated)), while the other kind of simplicity is a conceptual one: to conceive of and understand the notion of time without change. Which kind of simplicity is then to be preferred?

Again, I am not trying here to refute Shoemaker's argument, but rather to point out to difficulties that it needs to address, and to emphasize that these difficulties are related to the possibility of local freezes or to epistemological concerns - both of these being actually unnecessary if one is interested in the metaphysical issue of whether there can be time without change or not. Let us then separate the two 
(epistemological and metaphysical) claims, let us leave the epistemological one aside, and let us forget about the possibility of local freezes, since none of these claims really tackles the metaphysical question at hand. Let us then bear in mind Shoemaker's idea free from these two unnecessary burdens, and let us ask ourselves whether it is metaphysically possible and/or conceivable that there is a world where (global) temporal vacua occur, and what it means if it is. The most direct and simplest way to do this, I suggest, is to think of Shoemaker's thought-experiment simply as a brute metaphysical possibility: just suppose that there is a world W where global freezes sometimes happen, and where there can (allegedly) be time without change. ${ }^{1}$

Now, the point I want to get to is that even if we grant plausibility to such a brute metaphysical possibility claim, we are not out of trouble - but it is here that trouble gets really interesting.

The trouble in question is: if a global freeze occurs, what causes it to stop? That is, what causes changes in the world to start again, after a period when all change stopped for some time? Shoemaker himself saw this problem (Shoemaker (1969, p. 74-78)), and explicitly addressed it, but simply failed to follow it where it leads, since he wanted to be able to conclude that time without change is possible. Let us see this in more detail.

What can cause a global freeze to come to an end? It cannot be a sufficient cause existing at the instant that immediately precedes the end of a global freeze, since this instant is qualitatively exactly identical to all of the instants that are part of the freeze, and consequently, if it contained a sufficient cause for ending the freeze, the freeze would not last more than an instant - that is, there would be no freeze. Nor can it be an exterior cause, since by hypothesis a global freeze encompasses everything in the universe. It couldn't be anything that happened during the freeze either, since by hypothesis nothing (no change) happens during a global freeze. Shoemaker himself considered the possibility of "causality at a temporal distance" (Shoemaker (1969, p. 74-77), that is, a kind of causality where the cause and the effect are separated by a temporal distance-i.e. where the effect does not take place at the instant right after a sufficient cause does. But such a proposal is either mysterious, since it seems to violate without explanation our concept of what a sufficient cause is, or ... it brings us to what I take to be the interesting conclusion to draw from Shoemaker's thoughtexperiment (that he himself saw, but didn't want to draw (Shoemaker (1969, p. 78)), namely that the amount of time that elapsed is (at least a part of ${ }^{2}$ ) what causes the end of a global freeze. The reason why Shoemaker did not embrace this conclusion, that naturally follows from his own thought-experiment, is that it seems to imply something he wanted to reject, and explicitly did reject (without argument) from the very beginning of his article, namely that A-properties (he uses the label "McTaggartian

\footnotetext{
${ }^{1}$ When it comes to the controversy between relationism and substantivalism (absolutism) about time, it is often argued that only substantivalism, but not relationism, is compatible with a global freeze. I show this to be incorrect in Benovsky (2011). Both views are equally compatible with a period of global freeze.

${ }^{2}$ I mean : in addition to the state of the universe at the very last instant before a global freeze starts.
} 
properties") are genuine properties. The reasoning that seems to be involved here is that if mere passage of time is causally efficient, it means that $(i)$ properties like "having been in a state of a global freeze for $1 \mathrm{~h}$ " are causally efficient, (ii) that such properties are a kind of A-properties, and (iii) that if these are causally efficient, there is no ground to claim that they are not genuine properties. ${ }^{3}$

Shoemaker is far from being alone in thinking that A-properties are not causally efficient. Lewis (1976, p. 136) says that A-changes are mere 'Cambridge' changes and so not genuine changes at all; Mellor (1998), as most B-theorists, reduces Aproperties to B-properties and claims that "if B-facts [play the role of truthmakers for temporal beliefs], then A-facts do not, and if they do not, then they do not exist, since this is what they exist to do" (Mellor (1998, p. xi)); and in general all those who reject the existence of the A-series, following or being more or less vaguely inspired by McTaggart (1908), also obviously reject the A-properties' genuineness and causal powers.

Let us see if this reasoning is correct. To be more precise about the (alleged) acausality of the mere passage of time, Newton-Smith (1980, p. 29-34) distinguishes two cases:

(a) date causality that is usually rejected: "For instance, suppose some substance, S, is introduced into a flame and turns green. We would never dream of thinking that it turned green because it was put into a flame at just that time. To think that the date was causally relevant in this case is to take quite literally the idea that the time was ripe for change." (Newton-Smith (1980, p. 29))

and

(b) duration causality that is also usually rejected and that is the point at issue here : "We do not take seriously the suggestion that the mere passage of time is causally relevant to anything. If some object changes its state after having been in that state for some period of time, $t$, we would not think that its having been in that state for that period was causally sufficient for it to change. [...] Duration causality $[\ldots]$ is excluded ab initio as we cannot see how the mere passage of time could bring about a change in the state of the system." (Newton-Smith (1980, p. 29))

While I share Newton-Smith's concerns about date causality, I think that what he says about duration causality expresses a deeply-anchored prejudice that many hold against the notion of the passage of time. At least in the context of the debate about the possibility of time without change such a claim seems to be a mere prejudice - there just is no clear argument to the effect that it is not the passage of time that is causally responsible for the end of a period of a global freeze; indeed, as we have seen, other alternatives fail, and thus it seems to be the only way to account for it.

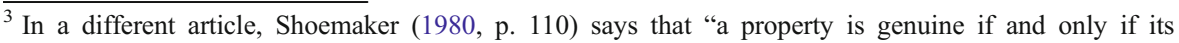
acquisition or loss by a thing constitutes a genuine change in that thing". In this definition, "genuine" lies on both sides of the biconditional, so it does not provide us with a good lead to understand why a property would count as genuine or not, unless it would beg the question against A-properties by claiming that they cannot be responsible for genuine change-which is precisely the point at issue.
} 
This then could be seen as a reductio not against the acausality of the passage of time, but rather against the very plausibility of a Shoemaker-like thought experiment. But, first, if one wants to avoid relying here only on a prejudice, and if one takes care to formulate a Shoemaker-like thought experiment in a way that avoids the aforementioned epistemological and local-freeze-related problems, it then seems that there just is no incoherence in the claim that there could be periods of time without change. As Newton-Smith (1980, p. 36) also remarks, it is true that duration causality looks quite unpalatable, but unpalatable doesn't mean incoherent or impossible, and, he suggests, it is perhaps precisely the consideration of Shoemaker-like scenarios that should lead us to accept duration causality as possible.

[A longish note : Perhaps there are actual cases of duration causality - that is, cases where it seems that mere passage of time is causally efficient. Cesium has 39 known isotopes, only one of which is stable, the others having usually a short radioactive period (fractions of a second to a couple of days). Thus, such a radioactive atom of Cesium will, after some time, decay. The point of interest for us now is that its decay is apparently not caused by anything - the disintegration is usually said by physicists to be 'spontaneous'. The decay of the atom is independent of any interactions, including any interactions with measurement devices or environmental conditions, it does not need outside triggering or manifestation conditions, but manifests itself spontaneously. There seems to be a large consensus among philosophers of science to claim that (a) the probability that an atom will decay is objective-i.e. it concerns nature, and not our knowledge-(b) it does apply to individual systems, and not only 'statistically' to systems of a number of atoms, and (c) it is a spontaneous process (see for instance Frigg and Hoefer (2007), and Dorato and Esfeld (2010)). It seems then to be the case that it is only the passage of time, and nothing else, that makes an individual atom decay. Thus, we have an atom in a certain state, and after some time, it changes its state, while no relevant condition other than the fact that some amount of time elapsed changed. This, I suggest, is analogous to a world W that is in a globally frozen state and that, after some time, changes its state (unfreezes) for no other reason than the fact that some amount of time elapsed. Furthermore, I suggest that this is a more plausible interpretation of this phenomenon than the one that David Lewis briefly offers in Lewis (1976, p. 140) where he says that the decay of the atom is uncaused. Uncaused events are mysterious and highly unpalatable, and one would never dream of asserting the existence of such a phenomenon - unless, again, one held a prejudice (or a philosophical argument) against causal efficiency of the passage of time.]

To come back to what I think should be the correct conclusion to draw from Shoemaker's thought-experiment, it is important to note that it would not be correct to say that if mere passage of time has causal powers, it would mean that A-properties are genuine and causally efficient (contrarily to what seems to be Shoemaker's worryand motivation for not following his argument where it leads). Indeed, a B-theorist could accept that what we learn from Shoemaker's thought experiment is that the mere fact that some amount of time elapsed between the beginning and the end of the freeze is what is responsible for the end of it, but she could interpret it in a Btheoretical way and say that it is the mere temporal distance (rather than some Aproperty) between the beginning and the end of a freeze that is causally efficient. 
Granted, the view that mere passage of time has causal powers is perhaps more natural under an A-theoretic reading : if there is something like A-theoretic genuine passage of time (and not just mere B-facts about temporal distance), one can more easily accept that it has causal powers - it is a real change in real and fundamental properties (A-change in genuine irreducible A-properties) that takes place in the world, and if this is the conception one has of temporal passage, then one does not have too many difficulties to attribute causal efficiency to it. On the other hand, the Btheorist, it seems, deprives temporal passage from any sort of 'genuine reality', reducing it to mere facts of temporal distance, and if this is the conception one has, then it does seem harder to claim that it has any causal powers. But, "harder" doesn't mean "impossible" and both the A and the B readings of my claim are acceptable.

Thus, if one takes care not to be simply prejudiced against the passage of time, then one may see a Shoemaker-like thought experiment as being a good reason to believe in (or as being a symptom of) its causal efficiency ${ }^{4,5}$

\section{References}

Benovsky, J. (2011). The relationist and substantivalist theories of time: foes or friends? European Journal of Philosophy, 19, $\mathrm{n}^{\circ} 4$.

Dorato, M., \& Esfeld, M. (2010). GRW as an ontology of dispositions. Studies in History and Philosophy of Modern Physics, 41, 41-49.

Frigg, R., \& Hoefer, C. (2007). Probability in GRW theory. Studies in History and Philosophy of Modern Physics, 38, 371-389.

Lewis, D. (1976). The paradoxes of time travel. First published in the American Philosophical Quarterly, 13, 145-152. Reprinted in Le Poidevin, R. and MacBeath M. (Eds.) 1993. The philosophy of time. Oxford University Press.

McTaggart, J. E. (1908). The unreality of time. Mind, 17, 457-474.

Mellor, D. H. (1998). Real time II. Routledge.

Newton-Smith, W. H. (1980). The structure of time. London: Routledge \& Kegam Paul.

Shoemaker, S. (1969). Time without change. First published in the Journal of Philosophy 66, 363-381. Reprinted in Le Poidevin, R. and MacBeath M. (Eds.) 1993. The philosophy of time. Oxford University Press.

Shoemaker, S. (1980). Causality and properties. In P. Van Inwagen (Ed.), Time and cause. Dordrecht.

Warmbrod, K. (2004). Temporal vacua. The Philosophical Quarterly, 54, n²15.

\footnotetext{
${ }^{4}$ One of the anonymous referees of this journal rightly stressed the important question concerning how the mere passage of time can be responsible for a release of a global freeze. Here, I think one might embrace an argumentative strategy close to Shoemaker's own concerning the very possibility of a global freeze. Indeed, Shoemaker does not provide a direct (positive) argument (reason) in favour of the possibility of a global freeze; rather his argumentative strategy is indirect: his argument is something like an argument from the best explanation. My strategy in this paper, regarding the possibility of causal efficiency of passage of time, is similar in this respect. The situation is the following: when there is a global freeze, there is nothing that can cause its end except the passage of time. The passage of time is the only thing that's left to account for the end of the freeze. Thus, I conclude: it must be it, since nothing else is available. In this way, I do not show how passage of time is responsible for the end of the global freeze, rather I show that it is the most reasonable assumption in the neighbourhood to suppose that it does so. This is then an indirect argumentative strategy as well.

${ }^{5}$ I would like to thank Jonathan Lowe for an insightful discussion about my first ideas for this article, as well as two anonymous referees and the Editor of this journal for very useful comments which helped me to improve the final version.
} 